\title{
BMJ Open Collaborative model of intrapartum care: qualitative study on barriers and facilitators to implementation in a private Brazilian hospital
}

\author{
Mercedes Colomar (D) , ${ }^{1}$ Franco Gonzalez Mora, ${ }^{2}$ Ana Pilar Betran (D) , ${ }^{3}$ \\ Newton Opiyo (D) , ${ }^{3}$ Meghan A Bohren (D) , ${ }^{4}$ Maria Regina Torloni (i) ,5, \\ Monica Siaulys ${ }^{5}$
}

To cite: Colomar M, Gonzalez Mora F, Betran AP, et al. Collaborative model of intrapartum care: qualitative study on barriers and facilitators to implementation in a private Brazilian hospital. BMJ Open 2021;11:e053636. doi:10.1136/ bmjopen-2021-053636

- Prepublication history and additional supplemental material for this paper are available online. To view these files, please visit the journal online (http://dx.doi.org/10.1136/ bmjopen-2021-053636).

Received 18 May 2021 Accepted 11 November 2021

Check for updates

(C) Author(s) (or their employer(s)) 2021. Re-use permitted under CC BY-NC. No commercial re-use. See rights and permissions. Published by BMJ.

For numbered affiliations see end of article.

Correspondence to Dr Maria Regina Torloni; torlonimr@gmail.com

\section{ABSTRACT}

Introduction A collaborative (midwife-obstetrician) model of intrapartum care (CMIC) is associated with lower caesarean section (CS) rates than physician-led models. In 2019, the largest private maternity hospital in Latin America (14.000 deliveries/year, 89\% CS) created a quality improvement initiative to optimise intrapartum care and safely reduce $\mathrm{CS}$ in low-risk women managed by its internal team of healthcare providers (HCP). We conducted formative research to identify potential barriers and facilitators to the implementation of a CMIC.

Methods Three groups of stakeholders participated in focus groups and interviews: hospital managers and clinical coordinators, HCP working in labour/delivery wards and pregnant women intending to give birth in the hospital. We explored participants' views about the acceptability of implementing a CMIC where a nurse-midwife (NM) on shift would be the main intrapartum HCP, with continuous support/supervision of a dedicated, in-house, obstetriciangynaecologist (OB-GYN). A thematic analysis approach was used.

Results 12 HCPs, 5 clinical coordinators, 2 hospital managers and 7 women participated. OB-GYNs, coordinators and managers highlighted health system, organisational and structural factors (NMs' limited experience/skills, professional roles, financial reimbursement) as potential barriers. NMs identified logistical and human resources as additional barriers. Women viewed the CMIC with perplexity and insecurity because of cultural beliefs about the dominant role of OBGYNs, and limited information about NM's capabilities. All professionals agreed that women's acceptance of a CMIC will require educational interventions and communication strategies to inform potential users about the advantages and safety of this model.

Conclusion There are important barriers and facilitators to implement a CMIC in a private Brazilian maternity hospital. Factors related to health system structure and organisation may have the greatest impact. A CMIC is more likely to succeed if stakeholders' concerns about responsibilities, power and financial revenues are addressed, and educational interventions targeted at users are deployed prior to its implementation.
Strengths and limitations of this study

- This is the first publication on barriers and facilitators to the implementation of a collaborative model (midwife-obstetrician) of intrapartum care in Brazil, a country with very high caesarean section rates.

- The inclusion of different types of participants (hospital administrators/managers, healthcare providers and pregnant women) allowed us to obtain different perspectives and to reach feasible and culturally tailored recommendations.

- This is the first qualitative study to assess the views of other key stakeholders, besides healthcare providers, on the implementation of this model of intrapartum care.

- The set of questions that we developed to guide the interviews and focus groups based on WHO generic protocol can be useful for other investigators who want to identify barriers and facilitators to the implementation of this model of care in their own settings.

- The findings of this study may not be transferable to all private maternities in Brazil or other countries.

\section{INTRODUCTION}

The proportion of women giving birth by caesarean section (CS) has increased substantially over the last decades, including in low-income and middle-income countries (LMICs) $){ }^{1-3}$ Part of this increase is due to CS performed for non-medical reasons. ${ }^{4}$ The situation has sparked international public health concern because of the lack of clinical benefits of unnecessary CS, increased maternal and perinatal short-term and long-term risks, and the diversion of human and financial resources, especially in LMICs. ${ }^{5-9}$ Unnecessary CS is a multifactorial problem that involves organisational, healthcare provider (HCP), user, community and cultural factors. Therefore, interventions to reduce unnecessary CS should be multifaceted, and ideally 
engage all stakeholders who contribute to this situation. 410

The model of care provided to women during labour and delivery is one of the organisational factors that can affect CS rates. ${ }^{11}$ In settings with physician-led care, labour and intrapartum interventions, including CS, tend to be higher than in other settings. ${ }^{11} 12$ In 2018, WHO recommended the collaborative model of care to reduce unnecessary $\mathrm{CS}$ in the context of rigorous research. ${ }^{13}$ In this model, nurse-midwives (NM) provide most obstetric care during labour and delivery for women managed in hospitals, with the support of a dedicated obstetriciangynaecologist (OB-GYN) who is continuously available in-house, without other competing professional activities. ${ }^{13}$ Given that changing models of care requires systems reorganisation-such as changes in professional roles and responsibilities and financial restructuring-formative research is essential to assess the local acceptability and feasibility of implementing the new model of care, and to adapt it to cultural and contextual factors. ${ }^{13} 14$ A recent systematic review ${ }^{15}$ found five qualitative studies ${ }^{16-20}$ that assessed stakeholder views about different staffing models to reduce unnecessary CS, but all were conducted in high-income countries.

Brazil is one of the countries with the highest CS rates in the world in the last decades. ${ }^{12}$ In 2018, the overall national CS rate was 56\%, reaching more than $80 \%$ in the private sector which is responsible for approximately $20 \%$ of all births each year. ${ }^{21} 22$ In Brazil, as in many countries, physicians are still the primary care providers for most pregnant women. In the Brazilian private sector (financed by health insurance companies or direct user payment), antenatal, intrapartum and postpartum care is typically provided by a single OB-GYN who usually works alone, without support from other healthcare professionals such as NMs. ${ }^{23}$ However, a proportion of Brazilian women with private health insurance receive antenatal care from an OB-GYN, but are managed during labour, birth, and the postpartum period by a team of HCPs on duty (internal team) at the hospital of their choice, whom they meet when they are admitted to give birth.

In 2018, there were 14468 livebirths at the Hospital e Maternidade Santa Joana (HMSJ) in São Paulo, Brazil, of which 12895 were by CS (overall CS rate of $89.1 \%$ ). ${ }^{24}$ In 2019, HMSJ created a quality improvement initiative to optimise the quality of intrapartum care and to safely reduce CS in low-risk women managed by its internal team. The implementation of a collaborative model of intrapartum care (CMIC) is one of the interventions included in this quality improvement initiative. Following the principles of implementation science for complex interventions and WHO recommendations, ${ }^{13} 14$ we conducted formative research to determine the local acceptability and feasibility of this organisational intervention, and to adjust the implementation plan according to these findings in order to optimise its effectiveness.

In this manuscript, we present the findings of the formative research conducted to identify and analyse the potential barriers and facilitators to the implementation of a collaborative (midwifery-obstetrician) model of intrapartum care in the largest private Brazilian maternity hospital.

\section{METHODS}

This study is reported according to the consolidated Criteria for Reporting Qualitative Research. ${ }^{25}$ The study conformed to the principles of the Declaration of Helsinki. All participants gave written informed consent. Patients and the public were not involved in the design, or conduct, or reporting, or dissemination plans of our research.

\section{Setting, design and participants}

This qualitative study was conducted in September 2019 at HMSJ, the largest private maternity hospital in Brazil, located in the city of São Paulo. In this tertiary-level centre for obstetrics and neonatal care, during labour and birth, pregnant women are cared for either by their own private physicians (external team), or by HCP on duty at the hospital (internal team) when they present for delivery. Approximately $70 \%$ of all deliveries at HMSJ are conducted by the external team and $30 \%$ by the internal team (OB-GYNs and NMs employed by the hospital). At the time of the study, the hospital's internal team consisted of three OB-GYNs and four NMs on 12-hour shifts working in a physician-led model of intrapartum care.

Three groups of stakeholders were included in this study: (1) hospital managers and clinical coordinators, (2) internal team of healthcare professionals (OBGYNs, anaesthetists and NMs working on regular shifts at and paid by the maternity hospital) and (3) pregnant women (of any parity, with and without previous CS) who intended to deliver at this hospital. We conducted focus groups with NMs and pregnant women, and semistructured in-depth interviews with the other participants. The managers set hospital policies and take care of financial aspects, while the clinical coordinators are in charge of the HCP teams and compliance with established healthcare procedures. At the time of the study, NMs helped doctors to manage women admitted for labour and delivery, provided non-pharmacological interventions to control pain, performed fetal monitoring and followed OB-GYNs' orders. The OB-GYNs were directly responsible for all intrapartum care, vaginal deliveries (VD) and CS; anaesthetists provided pharmacological analgesia for labour and $\mathrm{VD}$, and anaesthesia for CS. These groups were chosen to provide varying perspectives on the acceptability and feasibility of the implementation of a CMIC.

We purposively sampled managers, clinical coordinators, OB-GYNs and anaesthetists. For NMs and pregnant women, we used a convenience sample method. An administrative clerk who worked at the maternity hospital contacted potential participants by telephone and email 
Box 1 Theoretical scenario presented to participants about Models of Intrapartum Care

We are going to talk about "Models of Intrapartum Care" for low-risk women who are admitted in labour and managed by the team of healthcare providers on duty at the hospital (internal team) when they arrive. There are two basic ways (models) to take care of women who are admitted to a hospital to give birth: the "physician-led model of care" and the "collaborative model of care". (1) In the physician-led model, doctors are the main people in charge of caring for all healthy women throughout labour, and they attend all vaginal deliveries and caesareans, while nurse-midwives help with tasks such as checking vital signs, fetal heart rate monitoring, non-pharmacological pain relief and administrating medication prescribed by the doctor. (2) In the collaborative model, nurse-midwives (nurses with special training in obstetrics) are the main person in charge of caring for all healthy women throughout labour, and they attend all vaginal deliveries. But a doctor is nearby and available at all times, supporting and supervising the work of the nursemidwives, and ready to take appropriate action if and when needed. We want to understand what you think and how you feel about these Models of Intrapartum Care. When answering the questions, please imagine that both the woman and baby are healthy, that the woman intends to go into labour and to have a vaginal birth in this hospital managed by the healthcare provider team on duty.

inviting them to be part of the study. The interviews and focus groups were scheduled during participants' working hours. Pregnant women who were scheduled for one of the daily HMSJ hospital visits were contacted by the clerk by telephone and invited to participate in a focus group. Those who consented were invited to stay extra time at the end of their visit for the focus group which was conducted in the hospital. During these 30 min hospital visits which are regularly held twice daily, a group of 10-20 persons (pregnant couples and families who are planning to give birth at the hospital) are guided by a trained HMSJ clerk through relevant areas (admission, labour and delivery suites, postnatal rooms, neonatal unit).

The investigators presented to the participants a theoretical scenario of a new model of intrapartum care for low-risk women managed by the internal team of HCP where the NMs would play a more active role in providing obstetric care to these women, with the supervision and continued support of an OB-GYN on duty (box 1). The participants were asked about their views regarding this model of care, their willingness to accept it, and their opinion about its feasibility.

Participants completed a short demographic questionnaire. The interview and focus groups guidelines were adapted from the WHO generic formative research protocol designed as a guide to assess the local feasibility of and to tailor interventions to optimise the use of CS. ${ }^{14}$ Since this generic protocol does not have a specific section on collaborative model of care, we created the questions to guide the interviews and focus groups of this study (online supplemental file 1). Two experienced qualitative researchers (MC and FGM) who were not part of the maternity hospital staff conducted the field work, performed the analyses and prepared the final report. The researchers had never met any of the participants before. The sessions were moderated by one researcher while the other took notes and asked for clarification if needed. At the beginning of each session, the two investigators presented the objectives of the study, their credentials and background in gender and maternal health research, and asked participants to sign an informed consent form. The participants did not receive any financial compensation. Women received a snack and parking reimbursement for their time in the focus group. Each focus group lasted 60-90 min, and the individual interviews lasted about $60 \mathrm{~min}$. The sessions were conducted in quiet rooms at the maternity hospital, without any interruptions. Data collection continued until saturation was reached and no new information or themes emerged.

\section{Data analysis}

All sessions were audiorecorded for transcription, systematisation and analysis. The text transcripts were entered into Atlas-TI V.7. $0^{26}$ to organise the textual information. A combined inductive (themes emerging from the data) and deductive (themes based on the interview and focus group questions) thematic analysis approach was used. ${ }^{27}$ In order to improve the reliability of the study, the two members of the research team coded independently the transcripts according to the themes. Agreement was substantial; discrepancies were discussed until the researchers resolved them. The researchers categorised the themes and developed a matrix where the axis represented the codes used and the participants' categories. Finally, abstraction and interpretation of data was performed. As part of this analysis, we selected direct quotations representative of participants' opinions. Quotations were deidentified and translated from Portuguese to English for this article. Key informants among the study participants were selected to provide feedback and validate the findings (member checking).

For analyses, we organised the material taking into account three factors that affect the implementation of non-clinical interventions targeted at facilities to reduce unnecessary CS mapped in a 2018 systematic review: (1) health system, organisational and structural factors, (2) human and cultural factors and (3) mechanisms of effect to achieve change factors. Health system, organisational and structural factors capture how structural health systems, facility management and organisational factors that exist at an aggregate-level impact the values of stakeholders, and shape individual views of the feasibility, or otherwise, of interventions to reduce unnecessary CS. Human and cultural factors capture the way in which the culture in and of organisations may impact stakeholder views of interventions to reduce unnecessary CS. Finally, the mechanisms of effect to achieve change factors encompass the components that stakeholders identified as important to the implementation of interventions to reduce unnecessary CS. This theme builds on the previous two, in illustrating some of the mechanisms 
Table 1 Characteristics of focus group and interview participants, Hospital Maternidade Santa Joana, São Paulo, Brazil, 2019

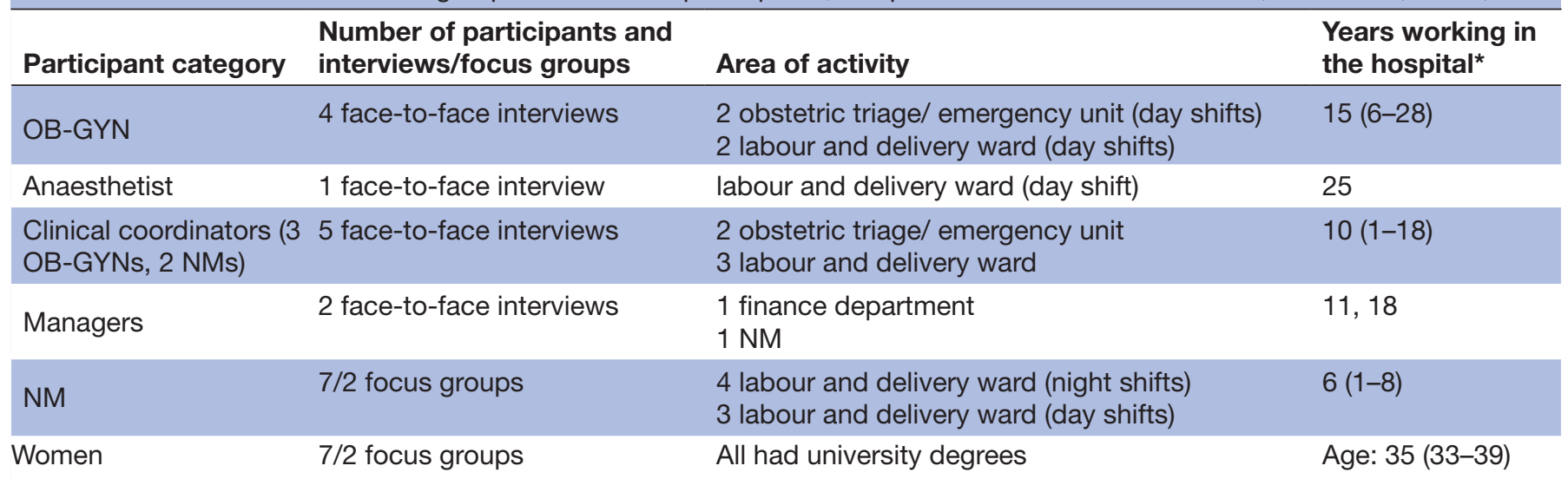

*Median (range).

NM, nurse-midwives; OB-GYN, obstetrician-gynaecologists.

to overcome entrenched relationships, and antagonistic cultural norms and behaviour. ${ }^{15}$ These factors were proposed by Kingdon et al based on a systematic qualitative review on-clinical interventions to reduce unnecessary CS targeted at organisations, facilities and systems ${ }^{15}$ which was included in the WHO recommendations. ${ }^{28}$ Kingdon et al used a modified meta-ethnography methodological approach which resulted in the three themes. The synthesis showed how intersystem and intrasystem power differentials, and stakeholder commitment, exert strong mechanisms of effect on CS rates, independent of the theoretical efficacy of specific interventions to reduce them. $^{15}$

\section{RESULTS}

The study involved 26 participants; box 1 presents their main characteristics. None of the hospital personnel refused the invitation to participate in the study. Seven out of 33 women scheduled for the daily regular hospital visits accepted to stay extra time to participate in the focus groups. We conducted two separate groups with these women to avoid long waiting periods, considering that there were two visits scheduled at different times on the same day. We conducted in-depth interviews with 12 individuals (4 OB-GYNs, 1 anaesthetist, 5 clinical coordinators and 2 managers) and 4 focus groups ( 2 with NMs and 2 with pregnant women) involving 14 persons (table 1 ).

\section{Views of OB-GYNs, clinical coordinators and managers about} a CMIC

We found agreement in the views of OB-GYNs, clinical coordinators and managers regarding the implementation of a CMIC. Most of the findings from these participants were related to barriers linked to health system, organisational and structural factors.

Participants raised concerns about the effects of this model of care on the traditional professional roles and power relationship between physicians and NMs. Most respondents expressed confidence in the skills, abilities, and professional competency of NMs to manage low-risk women during labour and birth. However, some OB-GYNs were worried because most NMs currently working in the hospital's labour and delivery wards were out of practice or lacked the necessary experience to manage vaginal births, since this is not part of their daily activities. An additional concern was that in the private sector, NMs are not perceived by women and their families as being competent and legitimate obstetric authorities who can be trusted to take decisions or to intervene during the birth process, and this may raise trust and credibility issues.

"I think that a NM can take charge (of intrapartum care). You don't need to have a doctor exclusively for this." (A clinical coordinator)

Participants also mentioned that the current model of payment of health insurance companies could be an important barrier to the CMIC. Clinical coordinators and managers informed that health insurance companies currently pay the hospital lower fees for VD performed by a NM than by an OB-GYN. Managers pointed that this financial model incentivises the hospital to support that physicians conduct all VDs, to avoid loss of revenue. On the other hand, OB-GYNs were reluctant to take full clinical responsibility for the VD of women who were going to be managed by NMs throughout labour, as they felt that the OB-GYN would not be well informed about these women's labour progress and any previous interventions.

"And who is going to be responsible for each patient? Because you can't put all the responsibility in the hands of the doctor, and let a nurse-midwife there (taking care of the woman), and the doctor doesn't even know what is going on. It's complicated." (An OB-GYN)

OB-GYNs also recognised women as key stakeholders to system change and raised concerns about women's acceptability of the proposed model of care that could be 
perceived as potentially threatening the receipt of high quality care. These OB-GYNs anticipated that women might consider that being mostly cared for by a NM could be perceived as lower quality care because, culturally, Brazilian women in private maternities expect and prefer physician-led care.

"I think that our Brazilian patient who comes to a private maternity like HMSJ does not want to be cared for by a nurse-midwife. She will think that this is suboptimal care; that's my impression. I think that this is a risk. I think that the patient needs to be informed that this is the way things work here: if you have a low-risk pregnancy and you come (to the maternity) to have a vaginal delivery, in principle, you will be cared for by a nurse-midwife. The woman has to be informed. Then, she can accept (this type of intrapartum care), or she can decide not to come (to this maternity)." (An OB-GYN)

The participants identified two mechanisms of effect for change factors targeted at women as important to implement the collaborative model: prenatal education, and a communication strategy about the benefits of CMIC. The information conveyed to women should emphasise that the CMIC is the "state of the art" model for good obstetric care, and that it is widely used in high income countries. The participants stated that one of the potential positive effects of implementing the CMIC would be the increase in the number of VD because NMs are typically less interventionists and foster a sense of trust and serenity to women in labour.

\section{Views of NMs about a CMIC}

NMs expressed concerns regarding professional roles, relationships, and power dynamics between physicians and NMs. Several NMs anticipated that some OB-GYNs would feel threatened by their new roles or would not trust their professional competencies, and these attitudes could be potential barriers to the implementation of the collaborative model. The NMs considered themselves qualified and to have the professional skills required to manage low-risk women during labour and delivery. However, they acknowledged that some of their colleagues currently working in the labour and delivery ward were not fully prepared, or lacked the necessary experience or confidence, to take on the more active role required of NMs in a CMIC.

The participants were confident that the hospital had all the resources needed to implement a CMIC and perceived the maternity's shared commitment to reduce CS rates.

Regarding the final responsibility for women's health and delivery outcomes in a CMIC, most NMs agreed that responsibility should be shared between NMs and OB-GYNs.

"I think that when you work in a team, and he (the doctor) is present all the time, he knows what is going on, and he trusts the work of the NM. I think that the NM will not run away from responsibility when the doctor takes over, she will be his partner. So, when it's time to sign (hospital documents), or to finalize a partogram, the doctor and the NM will both sign. Responsibility for the care of the patient will be shared by the doctor and the NM."

NMs pointed that organisational logistics could be a potential barrier for the implementation of a collaborative model of care because the increasing number of VD would require extra labour and delivery rooms, and more NMs to manage the larger number of labouring women.

NMs did not reach an agreement about their views of women's acceptability of a collaborative model. They predicted that while some women would refuse being cared for by a NM, others might feel more comfortable with this model of care, and a third group would be indifferent to the professional responsible for their care. However, given the current Brazilian model of physician-led obstetric care in the private sector, participating NMs thought that it will be challenging to increase their role and to implement a CMIC in this setting.

"The role of the doctor is more important. Because in this social class (of women managed at HMSJ), the doctor is much more valued. He is more visible, he stands on a pedestal, while the NM is (down) here, she is the woman's pal, her little friend."

Similar to the OB-GYNs, NMs thought that a communication strategy for women about the benefits of CMIC was an important mechanism of effect to achieve change factor. This communication or marketing strategy should clarify the role and importance of NMs in the CMIC, and that this model of care is the gold standard for low-risk women who want to have a safe and humanised childbirth. They predicted that after a transition period, women would accept the new model of care.

\section{Views of women about a CMIC}

Women were asked about the acceptability of a CMIC in which NMs would have a more active role in caring for low-risk women during labour and delivery. Their initial reaction was confusion and perplexity, mixed with a sense of insecurity and uncertainty regarding this new model of care due to unawareness that NM were professionals qualified to manage labour and delivery. After a process of reflection, the participants expressed different opinions; some women were receptive towards this model of care while others did not find it acceptable. These differences were rooted in each woman's preferred mode of delivery. We did not select women according to mode of birth preferences; therefore the focus groups included women who stated that they preferred an elective CS, women who preferred a vaginal birth, and those who did not have a clearly defined birth preference at the time. While the women who preferred a scheduled CS were sceptical about the collaborative model, those who wanted to have 
a vaginal birth were more favourable and open to a shift in the delivery care paradigm.

"...I would not mind having a NM deliver my baby."

"Me neither, as long as she showed me, at the time, that she had the necessary knowledge and that she was capable of providing a humanised birth too... Because the care that we receive also has to be the best."

Women's perceptions about the skills of NMs were rooted in their perspective of the traditional physician-led medicalised obstetric care offered in most private Brazilian maternities. The professional competences of NMs were largely ignored or not well understood, and they were viewed by the women as mere assistants to the OB-GYNs. Some women were open to be cared for by a NM during labour and birth, as long as her work was directly supervised by an OB-GYN who was nearby and immediately available to intervene in case of need. On the other hand, all women valued the role of NMs in providing respectful, humanised care and emotional support during labour and birth.

"I think that, whether you want to or not, you have a lot of confidence in what doctors say. (....... it's something that is embedded in people's heads, it is associated with safety and care. A NM, in my opinion, is something like... a companion to the doctor, but she is not the doctor. So, I think that there are situations where it is OK to have a NM (taking care of women). For instance, if I arrived and I'm giving birth outside (of the labour ward), or there isn't enough time to go up, then it's great to have someone there to help me; it could be a fireman, it could be anyone. Or if I am (giving birth) in a taxi...it could be the cab driver; as long as everything went well, if everything was OK. But if I have the choice of being in a hospital, I would really like the option to have a doctor (taking care of me)."

Table 2 summarises the main barriers and facilitators for the implementation of the CMIC derived from the interviews and focus group discussions with hospital personnel and women, stratified according to the three levels proposed by Kingdon et al. ${ }^{15}$

\section{DISCUSSION}

This study looked at the factors affecting the feasibility and acceptability of implementing a CMIC, a model that challenges the current intrapartum care paradigm in most private Brazilian hospitals. We found a strong agreement in the views and perspectives of different stakeholders on the critical factors that may influence the implementation of a collaborative midwifery-obstetrician model of intrapartum care. HCPs thought that factors related to the health system structure and organisation would have the greatest impact on the implementation of this strategy.
NMs working in a CMIC need to be trusted, respected and supported by whole hospital staff, including OB-GYNs, hospital managers and directors. ${ }^{29-31}$ Although most HCPs believed that NMs had the required skills to manage low-risk women during labour and delivery, some physicians and NMs raised concerns about the competence and lack of experience of some individuals. Similarly, a 2018 systematic review on organisational barriers and facilitators to the implementation of non-clinical interventions to reduce unnecessary CS reported that in hospitals/facilities implementing midwifery led care models, there was dissatisfaction from doctors who felt their professional identity and the safety of women was compromised by relinquishing lead professional responsibility to midwives. ${ }^{15}$ All HCPs agreed on the importance of ensuring that the NMs who participate in the CMIC are adequately trained and have sufficient experience to provide high-quality obstetric care to labouring women, and pointed to the potential risks and threats to sustainability if this is not guaranteed. There is increasing evidence that poor midwifery education and lack of practical experience are important barriers to the provision of quality midwifery care in low- and middle-income countries. ${ }^{32}$

Since skilled and experienced NMs are essential in this model of care, hospital managers will need to revise the skillsets required when hiring new NMs to work in the labour and delivery wards of private hospitals, and potentially upskill current NM employees through in-service training. However, NMs competencies in providing good intrapartum care to private patients will only be valued when the delivery care paradigm changes. As pointed by some participants, efforts to reduce unnecessary CS by implementing a CMIC should also take into account the potential logistics and human resources barriers that will naturally appear as the number of VD increase. The hospital must be prepared to provide more private labour and delivery rooms to accommodate the increasing number of women who will require longer time to give birth vaginally, and hire more NMs to manage these women, leading to increased financial costs. However, in the future, these changes in the institution's infrastructure and human resources to fully implement a CMIC could lead the maternity hospital to be seen as a reference centre in the private sector for safe vaginal births of low-risk women who are not managed by their own private physicians.

Current differences in payments for VD by health insurance companies according to the professional in charge of delivery incentivise that OB-GYNs conduct all vaginal births, leading to potential conflicts in the roles of NMs and physicians regarding who should assist the delivery. OB-GYNs expressed worries about having to take full clinical responsibility for the delivery of women who had been mostly managed by NMs in a collaborative model, to avoid loss of hospital revenue. A reform in the current payment system for VDs by insurance companies could facilitate the implementation of a collaborative model 
Table 2 Stakeholders' views of the main barriers and facilitators to implement a collaborative model of intrapartum care at a private maternity hospital in Brazil

\begin{tabular}{lll}
$\begin{array}{l}\text { Barriers related to health system, } \\
\text { organisational and structural factors }\end{array}$ & $\begin{array}{l}\text { Barriers related to human and cultural } \\
\text { factors }\end{array}$ & $\begin{array}{l}\text { Mechanisms of effect to achieve } \\
\text { change factors }\end{array}$ \\
\hline
\end{tabular}

\section{Views of physicians, clinical coordinators and hospital managers}

Belief that the university curriculum and Perception that some NMs do not have the professional training of Brazilian NMs is deficient and therefore they are not prepared to manage labour and delivery

\section{The current financial model of insurance} companies incentivises all vaginal deliveries be conducted by OB-GYNs will not perceive NMs as credible obstetric necessary skills and experience, and lack confidence, to assist women during labour and delivery OB-GYNs predict that some women/families authorities and will perceive management by a NM during labour and delivery as lower quality of care
OB-GYNs are reluctant to take full clinical responsibility for the vaginal delivery of women who were managed by NMs during labour because of insurance companies' financial model

Reassure OB-GYNs they will not lose their jobs as NMs assume a more active role during labour and delivery

Most medical staff need to believe that NMs have adequate technical abilities and skills to manage the labour and delivery of low-risk women

Disseminate information to potential users that the hospital has a trained team of dedicated NMs and OB-GYNs who work collaboratively to ensure a safe and humanised birth experience

Involve OB-GYNs in the promotion and implementation of the collaborative model of care

Designate a specific OB-GYN for each woman managed by a NM. Even if this physician is not permanently present during labour and at delivery, it would increase women's acceptability of being cared by a NM

\section{Views of nurse midwives}

As the number of vaginal deliveries increase, Perception that some NMs do not have the the hospital will need to create more private required skills, training, experience, and labour and delivery rooms and hire more NMs for all shifts. This will increase costs for and vaginal delivery the hospital
Promote and foster a climate of trust and respect for the work and competence of NMs within the institution and in all multiprofessional teams

Perception that some OB-GYNs do not trust
NMs' competence to care for women during
labour and delivery

Some women will not accept being taken care of by a NM in a private hospital

Perception that some OB-GYNs do not trust Grant greater autonomy to NMs and encourage those who are better trained to start taking care of low-risk vaginal deliveries

Increase the participation of NMs in antenatal care and in the emergency/ obstetric triage department to establish a relationship with women before their admission in labour, and to foster their trust in the technical competence of NMs

Assure that OB-GYNs are quickly available when NMs ask for support or when complications arise. NMs need to know that they can count on this safety net when needed in the management of lowrisk women

Clear definitions of NMs' and OB-GYNs' professional, administrative, and financial responsibilities in the collaborative model of care

Views of Women

The women who preferred a scheduled CS Women willing to have a vaginal birth were were sceptical about the collaborative model more favourable and open to a shift in the delivery care paradigm 
Table 2 Continued

\begin{tabular}{lll}
\hline $\begin{array}{l}\text { Barriers related to health system, } \\
\text { organisational and structural factors }\end{array}$ & $\begin{array}{l}\text { Barriers related to human and cultural } \\
\text { factors }\end{array}$ & $\begin{array}{l}\text { Mechanisms of effect to achieve } \\
\text { change factors }\end{array}$ \\
\hline & $\begin{array}{l}\text { NM capabilities were largely ignored by } \\
\text { women }\end{array}$ & $\begin{array}{l}\text { All women valued the role of NMs in } \\
\text { providing respectful, humanised care } \\
\text { and emotional support during labour and } \\
\text { delivery }\end{array}$ \\
\hline
\end{tabular}

CS, caesarean section; NM, nurse-midwife; OB-GYN, obstetrician-gynaecologist.

of care and the reduction of CSs in the private sector in Brazil. This is currently being discussed between the national agency that regulates the private health sector and the association of health insurance companies.

All participants mentioned the Brazilian cultural expectation that an OB-GYN be present in all vaginal births in private hospitals, and how this could be an important barrier to the implementation of a CMIC. This can be relevant in settings where private maternities compete to attract potential customers by tying to fulfil their perceived needs. This cultural factor could be crucial to the success or failure of the implementation of this model of care, and may require communication campaigns to change established stereotypes and views. Mass media campaigns have been recommended to change population health behaviours ${ }^{33}$ and may be a useful approach to address these fears. HCPs, managers and coordinators in our study suggested educational interventions during antenatal care, or targeting women who visit the hospital, to explain the competence and role of NMs in providing good quality and safe intrapartum care and to present the collaborative model as the gold standard of care for humanised vaginal births in low-risk women. There is evidence to support educational interventions to reduce unnecessary $\mathrm{CS},{ }^{435}$ and these could help to establish a basis for more informed and meaningful dialogues between women and HCPs. While we wait for the effects of these educational strategies and a payment reform, a possible solution is to continue having an OB-GYN present at all VD, working in close collaboration with the NM who managed the woman throughout labour.

A positive response to this model could be anticipated because of the growing number of Brazilian women who are looking for less medicalised intrapartum care, and who value the more comforting and empathetic care provided by NMs. According to a recent study conducted in a public hospital in southern Brazil, women managed by NMs during labour and delivery perceived them as important in providing non-pharmacological pain relief, emotional support and respectful, humanised care. ${ }^{36}$ Our pregnant participants mentioned exactly the same attributes as reasons for valuing the work of NMs.

Studies in high-income countries also reported that NMs had concerns about the limited trust in their skills and competence, and the lack of support for their role in some organisations and systems. ${ }^{16171937}$ Strategies that promote a greater involvement of NMs in a CMIC seem to be an adequate response to the need to 'demedicalise' low-risk births which represent most of the obstetric population.

While in many countries NMs are the primary HCP during labour and perform most VD, the participation of NMs in intrapartum care is limited in Brazil. ${ }^{38-40}$ According to a large study involving 266 Brazilian hospitals and nearly 11 500 vaginal births, NMs conducted only $7.2 \%$ of VD in the private sector. ${ }^{41}$ The same study reported that $\mathrm{CS}$ rates were significantly lower in settings where NMs were part of the labour care team than in physician-led care settings $(41.4 \%$ vs $58.4 \%$, respectively), illustrating the potential benefits of collaborative obstetric care in Brazilian hospitals.

This study had several strengths. The data were coded and classified into categories by two researchers to limit potential biases and inappropriate interpretation of the transcripts. Moreover, throughout the analytical process, the findings were checked with representatives of the study population. The inclusion of different types of participants allowed us to obtain different perspectives and to reach feasible and culturally tailored recommendations. Previous studies have assessed views of HCPs on the implementation of midwife-led models of care ${ }^{18}{ }^{19}$ However, we did not identify other qualitative studies that included the views of other key stakeholders about the implementation of this model of care. Finally, this is the first publication on barriers and facilitators to the implementation of a CMIC in Brazil.

We acknowledge that the findings of this study may not be transferable to all private maternities in Brazil or other countries. However, they could be applicable to similar, large private institutions that also have high CS rates, and this analysis is an important starting point to address the very high CS rates in private settings. The set of questions that we developed to guide the interviews and focus groups based on the WHO generic protocol can be useful for other investigators who want to identify barriers and facilitators to the implementation of a CMIC in their own settings. These questions can also be useful for future updates of the generic protocol created by WHO. ${ }^{14}$

\section{CONCLUSION}

We identified important barriers and facilitators to the implementation of a CMIC in a large private maternity hospital. Factors related to the health system structure and organisation may have the greatest impact on the implementation of this model of care. The CMIC is more likely to succeed if stakeholders' concerns about 
responsibilities, workload, power and financial revenues are addressed, and if educational interventions targeted at women are deployed prior to its implementation.

\section{Author affiliations}

${ }^{1}$ Montevideo Clinical and Epidemiological Research Unit, Montevideo, Uruguay ${ }^{2}$ Health Sociology Unit, University of the Republic of Uruguay Faculty of Medicine, Montevideo, Uruguay

${ }^{3}$ UNDP/UNFPA/UNICEF/WHO/World Bank Special Programme of Research, Development and Research Training in Human Reproduction, Department of Sexual and Reproductive Health and Research, World Health Organization, Geneva, Switzerland

${ }^{4}$ Gender and Women's Health Unit, Centre for Health Equity, Melbourne School of Population and Global Health, University of Melbourne, Melbourne, Victoria, Australia ${ }^{5}$ Hospital e Maternidade Santa Joana, Sao Paulo, Brazil

Contributors MRT, MS and APB conceptualised and designed the study. MC and FGM conducted data acquisition. MC, FGM, MRT, MS, NO, MAB and APB participated in data analysis and interpretation. MRT, MC and FGM wrote the original draft. MS, NO, MAB and APB revised the manuscript and made substantial critical contributions. MRT, MC, FGM, MS, NO, MAB and APB approved the manuscript version submitted to the journal. MRT, MC, FGM, MS, NO, MAB and APB are accountable for all aspects of the work in ensuring that questions related to the accuracy or integrity of any part of the work are appropriately investigated and resolved. MS accepts full responsibility for the work and/or the conduct of the study, had access to the data, and controlled the decision to publish.

Funding This study was funded by Hospital e Maternidade Santa Joana, São Paulo, Brazil.

Competing interests None declared.

Patient consent for publication Not applicable.

Ethics approval Hospital e Maternidade Santa Joana 3.596.366.

Provenance and peer review Not commissioned; externally peer reviewed.

Data availability statement No data are available. The transcripts of the focus groups and in-depth interviews are not available due to confidentiality issues.

Supplemental material This content has been supplied by the author(s). It has not been vetted by BMJ Publishing Group Limited (BMJ) and may not have been peer-reviewed. Any opinions or recommendations discussed are solely those of the author(s) and are not endorsed by BMJ. BMJ disclaims all liability and responsibility arising from any reliance placed on the content. Where the content includes any translated material, BMJ does not warrant the accuracy and reliability of the translations (including but not limited to local regulations, clinical guidelines, terminology, drug names and drug dosages), and is not responsible for any error and/or omissions arising from translation and adaptation or otherwise.

Open access This is an open access article distributed in accordance with the Creative Commons Attribution Non Commercial (CC BY-NC 4.0) license, which permits others to distribute, remix, adapt, build upon this work non-commercially, and license their derivative works on different terms, provided the original work is properly cited, appropriate credit is given, any changes made indicated, and the use is non-commercial. See: http://creativecommons.org/licenses/by-nc/4.0/.

\section{ORCID iDs}

Mercedes Colomar http://orcid.org/0000-0001-7424-5551

Ana Pilar Betran http://orcid.org/0000-0002-5631-5883

Newton Opiyo http://orcid.org/0000-0003-2709-3609

Meghan A Bohren http://orcid.org/0000-0002-4179-4682

Maria Regina Torloni http://orcid.org/0000-0003-4944-0720

\section{REFERENCES}

1 Betrán AP, Ye J, Moller A-B, et al. The increasing trend in caesarean section rates: global, regional and national estimates: 1990-2014. PLoS One 2016;11:e0148343.

2 Boerma T, Ronsmans C, Melesse DY, et al. Global epidemiology of use of and disparities in caesarean sections. Lancet 2018;392:1341-8.

3 Zhao Y, Zhang J, Zamora J, et al. Increases in caesarean delivery rates and change of perinatal outcomes in low- and middle-income countries: a hospital-level analysis of two who surveys. Paediatr Perinat Epidemiol 2017;31:251-62.

4 Betrán AP, Temmerman M, Kingdon C, et al. Interventions to reduce unnecessary caesarean sections in healthy women and babies. Lancet 2018;392:1358-68.

5 Keag OE, Norman JE, Stock SJ. Long-term risks and benefits associated with cesarean delivery for mother, baby, and subsequent pregnancies: systematic review and meta-analysis. PLoS Med 2018;15:e1002494.

6 Liu S, Liston RM, Joseph KS, et al. Maternal mortality and severe morbidity associated with low-risk planned cesarean delivery versus planned vaginal delivery at term. CMAJ 2007;176:455-60.

7 Sandall J, Tribe RM, Avery L, et al. Short-term and long-term effects of caesarean section on the health of women and children. Lancet 2018;392:1349-57.

8 Sobhy S, Arroyo-Manzano D, Murugesu N, et al. Maternal and perinatal mortality and complications associated with caesarean section in low-income and middle-income countries: a systematic review and meta-analysis. Lancet 2019;393:1973-82.

9 Souza JP, Gülmezoglu A, Lumbiganon P, et al. Caesarean section without medical indications is associated with an increased risk of adverse short-term maternal outcomes: the 2004-2008 who global survey on maternal and perinatal health. BMC Med 2010;8:71.

10 Visser GHA, Ayres-de-Campos D, Barnea ER, et al. Figo position paper: how to stop the caesarean section epidemic. Lancet 2018;392:1286-7.

11 Chapman A, Nagle C, Bick D, et al. Maternity service organisational interventions that AIM to reduce caesarean section: a systematic review and meta-analyses. BMC Pregnancy Childbirth 2019;19:206

12 Sutcliffe K, Caird J, Kavanagh J, et al. Comparing midwife-led and doctor-led maternity care: a systematic review of reviews. J Adv Nurs 2012;68:2376-86.

13 World Health Organization. WHO recommendations on non-clinical interventions to reduce unnecessary caesarean sections (Licence: CC BY-NC-SA 3.0 /GO). Geneva: World Health Organization, 2018.

14 Bohren MA, Opiyo N, Kingdon C, et al. Optimising the use of caesarean section: a generic formative research protocol for implementation preparation. Reprod Health 2019;16:170.

15 Kingdon C, Downe S, Betran AP. Non-clinical interventions to reduce unnecessary caesarean section targeted at organisations, facilities and systems: systematic review of qualitative studies. PLoS One 2018;13:e0203274.

16 Cheyne H, Abhyankar P, McCourt C. Empowering change: realist evaluation of a Scottish government programme to support normal birth. Midwifery 2013;29:1110-21.

17 Hunter B. Implementing a national policy initiative to support normal birth: lessons from the all Wales clinical pathway for normal labour. $J$ Midwifery Womens Health 2010;55:226-33.

18 Hunter B, Segrott J. Using a clinical pathway to support normal birth: impact on practitioner roles and working practices. Birth 2010;37:227-36.

19 Hunter B, Segrott J. Renegotiating inter-professional boundaries in maternity care: implementing a clinical pathway for normal labour. Sociol Health IIIn 2014;36:719-37.

20 Sakala C. Midwifery care and out-of-hospital birth settings: how do they reduce unnecessary cesarean section births? Soc Sci Med 1993;37:1233-50.

21 Agência Nacional de Saúde Suplementar (ANS) 2019. Taxas de partos cesáreos por operadora de plano de saúde [Internet]. BRASIL, ANS, 2018: 1-6. http://www.ans.gov.br/16-planos-de-saude-eoperadoras/informacoes-e-avaliacoes-de-operadoras/5063-taxaspartos-cesareos-brasil-2018

22 Ministério da Saúde, DATASUS/SINASC. [Livebirths Information System of the Brazilian Ministry of Health]. [Internet], 2018. Available: http://tabnet.datasus.gov.br/cgi/deftohtm.exe?sinasc/cnv/nvuf.def

23 Torres JA, Leal MdoC, Domingues RMSM, et al. Evaluation of a quality improvement intervention for labour and birth care in Brazilian private hospitals: a protocol. Reprod Health 2018;15:194.

24 Ministério da Saúde, DATASUS/SINASC, Painel de Monitoramento de Nascidos Vivos. [Hospital Monitoring Panel, Livebirths Information System of the Brazilian Ministry of Health] [Internet], 2018. Available: http://svs.aids.gov.br/dantps/centrais-de-conteudos/paineis-demonitoramento/natalidade/grupos-de-robson/

25 Tong A, Sainsbury P, Craig J. Consolidated criteria for reporting qualitative research (COREQ): a 32-item checklist for interviews and focus groups. Int J Qual Health Care 2007;19:349-57.

26 Muhr T, Atlas TI. berlin: "Scientific Software Development", 2020.

27 Braun V, Clarke V. Using thematic analysis in psychology. Qual Res Psychol 2006;3:77-101. 
28 Opiyo N, Kingdon C, Oladapo OT, et al. Non-clinical interventions to reduce unnecessary caesarean sections: who recommendations. Bull World Health Organ 2020;98:66-8.

29 Stevens JR, Witmer TL, Grant RL, et al. Description of a successfu collaborative birth center practice among midwives and an obstetrician. Obstet Gynecol Clin North Am 2012;39:347-57.

30 Chodzaza E, Mbiza C, Gadama L, et al. Midwives' and medical professionals' perspectives of collaborative practice at Queen Elizabeth central Hospital maternity unit, Malawi: the discovery phase of an appreciative inquiry project. Malawi Med J 2020;32:13-18.

31 Downe S, Finlayson K, Fleming A. Creating a collaborative culture in maternity care. J Midwifery Womens Health 2010;55:250-4.

32 Filby A, McConville F, Portela A. What prevents quality midwifery care? A systematic mapping of barriers in low and middle income countries from the provider perspective. PLoS One 2016;11:e0153391.

33 Torloni MR, Brizuela V, Betran AP. Mass media campaigns to reduce unnecessary caesarean sections: a systematic review. BMJ Glob Health 2020;5:e001935.

34 Wakefield MA, Loken B, Hornik RC. Use of mass media campaigns to change health behaviour. Lancet 2010;376:1261-71.

35 Kingdon C, Downe S, Betran AP. Women's and communities' views of targeted educational interventions to reduce unnecessary caesarean section: a qualitative evidence synthesis. Reprod Health 2018;15:130.

36 Lima MM, Ribeiro LN, Costa R, et al. Enfermeiras obstétricas no processo de parturição: percepção das mulheres [Obstetric nurses in the childbirth process: the women's perception] [Enfermeras obstétricas en el proceso del parto: percepción de las mujeres]. Revista Enfermagem UERJ; v 28 (2020)DO - 1012957/ reuerj202045901 2020.

37 Behruzi R, Hatem M, Fraser W, et al. Facilitators and barriers in the humanization of childbirth practice in Japan. BMC Pregnancy Childbirth 2010;10:25.

38 Carr M, Riesco MLG. Rekindling of nurse-midwifery in Brazil: public policy and childbirth trends. J Midwifery Womens Health 2007;52:406-11.

39 Gualda DMR, Narchi NZ, de Campos EA. Strengthening midwifery in Brazil: education, regulation and professional association of midwives. Midwifery 2013;29:1077-81.

40 Narchi NZ. Exercise of essential competencies for midwifery care by nurses in São Paulo, Brazil. Midwifery 2011;27:23-9.

41 Gama SGNda, Viellas EF, Torres JA, et al. Labor and birth care by nurse with midwifery skills in Brazil. Reprod Health 2016;13:123. 